\title{
Étude descriptive des tests de dépistage de la syphilis au Manitoba, Canada, 2015 à 2019
}

\author{
Souradet Shaw ${ }^{1 \star}$, Pierre Plourde ${ }^{2,3}$, Penny Klassen ${ }^{4}$, Derek Stein ${ }^{3,4}$
}

\section{Résumé}

Contexte : En 2018, le Manitoba affichait le plus haut taux déclaré de syphilis infectieuse au Canada, soit plus de trois fois la moyenne nationale. Dans la province, la syphilis infectieuse touche principalement les jeunes couples hétérosexuels marginalisés du centre-ville de Winnipeg. Par la suite, une crise de santé publique découlant de la syphilis congénitale est apparue au Manitoba, juste avant la pandémie de maladie à coronavirus 2019 (COVID-19). Le dépistage de la syphilis (dans le cas d'une grossesse) est considéré comme une mesure efficace pour réduire l'incidence de la syphilis et de ses séquelles. L'objectif de cette étude est de décrire les pratiques de dépistage de la syphilis dans la population générale et chez les femmes enceintes pendant une épidémie de syphilis.

Méthodes : Les données d'analyse utilisées de la syphilis fondées sur la population provenaient du laboratoire provincial Cadham (Winnipeg, Manitoba) pour la période de 2015 à 2019. Les taux normalisés selon l'âge sont déclarés directement, et la régression de Poisson a été utilisée pour modéliser les déterminants des taux de dépistage. Les taux de dépistage prénatal sont également déclarés.

Résultats : De 2015 à 2019, un total de 386350 personnes ont subi un test de dépistage de la syphilis. Le taux a augmenté chaque année, passant de 462 pour 10000 habitants en 2015 à 704 pour 100000 en 2019, tandis que le ratio femme-homme a diminué, passant de 1,8 à 1,6. Avant 2019, la majorité des femmes enceintes (environ 60 \%) ont fait l'objet d'un dépistage une fois, au cours du premier trimestre; toutefois, en 2019, davantage de femmes ont subi plus de deux tests au cours de leur grossesse.

Conclusion : Il y a eu une augmentation globale du nombre de personnes testées, ce qui reflète le taux accru de syphilis au Manitoba. Les tendances du dépistage prénatal ont changé en 2019, probablement en raison de l'augmentation du nombre de cas de syphilis congénitale.
Cette oeuvre est mise à la disposition selon les termes de la licence internationale Creative Commons Attribution 4.0

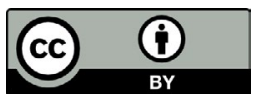

\begin{abstract}
Affiliations
${ }^{1}$ Institut de santé publique mondiale, Département des sciences de la santé communautaire, Université du Manitoba, Winnipeg, MB

${ }^{2}$ Santé publique et des populations, Office régional de la santé de Winnipeg, Winnipeg, MB

${ }^{3}$ Département de microbiologie médicale, Université du Manitoba, Winnipeg, MB

${ }^{4}$ Laboratoire provincial Cadham, Winnipeg, MB
\end{abstract}

\section{*Correspondance : \\ souradet.shaw@umanitoba.ca}

Citation proposée : Shaw SY, Plourde PJ, Klassen P, Stein D. Étude descriptive des tests de dépistage de la syphilis au Manitoba, Canada, 2015 à 2019. Relevé des maladies transmissibles au Canada 2022;48(2/3):107-14. https://doi.org/10.14745/ccdr.v48i23a07f

Mots-clés : syphilis, dépistage, soins prénataux, surveillance

\section{Introduction}

Au cours de la dernière décennie, l'Amérique du Nord a connu une augmentation du fardeau de la syphilis infectieuse, I'infection transmise sexuellement causée par la bactérie Treponema pallidium (T. pallidium) (1-3). Entre 2014 et 2018, les taux bruts de syphilis infectieuse au Canada ont augmenté de $153 \%$, passant de 7 à 17 pour 100000 habitants (4). De même, aux États-Unis, le nombre total de cas de syphilis infectieuse en 2018 ( $N=35063$ ) était le plus élevé observé depuis 1991, pour un taux de 11 pour 100000 habitants; il s'agit d'une augmentation de $70 \%$ depuis 2014 (2). La résurgence de la syphilis infectieuse a d'abord été observée principalement chez les gais, les bisexuels et les autres hommes ayant des rapports sexuels avec des hommes (gbHARSAH) à l'échelle mondiale. L'épidémie s'est ensuite propagée aux populations hétérosexuelles marquées par des problèmes structurels tels que la consommation de substances, l'incarcération et la pauvreté. Cette situation s'est traduite par une augmentation notable des infections chez les femmes $(2,5)$, ce qui pose de sérieux défis à la santé publique en termes de prévention, d'intervention et de suivi $(5,6)$. 
Les séquelles de la syphilis comprennent la neurosyphilis, qui peut être présente à n'importe quel stade de l'infection $(4,7)$, et la syphilis congénitale, qui peut avoir des conséquences catastrophiques pour le nourrisson et qui peut également se produire chez les femmes enceintes (8-10). Toute occurrence de syphilis congénitale dans les pays à revenu élevé est considérée comme un événement sentinelle et comme une " tragédie inutile » $(8,11)$. On a observé une augmentation des cas de syphilis congénitale en Amérique du Nord $(2,4,12,13)$, car I'incidence de la syphilis congénitale est associée à la prévalence de la syphilis infectieuse chez les femmes en âge de procréer (2).

Les données probantes appuient le dépistage des femmes enceintes comme méthode très efficace et rentable pour réduire l'incidence de la syphilis congénitale $(14,15)$. Le dépistage des populations adultes et des adolescentes qui ne sont pas enceintes, mais vulnérables à la syphilis, a également été recommandé (16). Dans les populations vulnérables, la modélisation mathématique a montré que les programmes de dépistage de la syphilis à couverture et à intensité élevées peuvent réduire l'incidence (17), tandis que le dépistage sousoptimal de la syphilis a été associé à une incidence d'équilibre plus élevée (18). Des études ont démontré l'existence de taux élevés de dépistage prénatal dans les pays à revenu élevé, comme les États-Unis. Toutefois, ces taux élevés de dépistage ne sont pas nécessairement applicables à des populations plus larges (non assurées) ou à d'autres pays (19). Par conséquent, le dépistage demeure un outil recommandé dans l'approche de santé publique contre la syphilis. II existe cependant un manque d'études fondées sur la population relativement aux taux réels de dépistage de la syphilis, et sur la façon dont les taux de dépistage peuvent avoir changé en réponse à l'épidémie croissante de syphilis.

En 2018, le Manitoba a déclaré le plus haut taux de syphilis infectieuse au Canada, soit 61 pour 100000 habitants (4), et la province était en voie d'afficher le plus grand nombre de cas de syphilis congénitale (20). Compte tenu de l'absence d'études représentatives sur le dépistage, des taux de dépistage de la syphilis, de l'augmentation des cas de syphilis infectieuse et des préoccupations au sujet des séquelles, cette étude visait à décrire les pratiques de dépistage de la syphilis dans la population générale et chez les femmes enceintes durant une recrudescence importante des cas de syphilis.

\section{Méthodes}

\section{Source de données}

Tous les tests de syphilis au Manitoba sont effectués au laboratoire provincial de Cadham. Le dépistage des infections transmissibles sexuellement et par le sang est offert gratuitement aux Manitobains (21). Le laboratoire provincial Cadham utilise un algorithme inversé pour le dépistage et le diagnostic de routine de la syphilis. Le test consiste en un essai par chimiluminescence initial pour les anticorps lgG et lgM contre les antigènes de tréponèmes. Les résultats positifs sont testés de façon plus approfondie au moyen de tests quantitatifs de dépistage de la syphilis infectieuse avec anticorps réaginique ou des tests du Laboratoire de recherche sur les maladies vénériennes. Les nouveaux cas positifs font l'objet d'un test d'agglutination passive de T. pallidum aux fins de confirmation. Le Manitoba offre également un programme de dépistage prénatal, qui comprend un dépistage sérologique d'un certain nombre d'infections transmissibles sexuellement et par le sang.

En réponse au nombre croissant de cas de syphilis congénitale, les lignes directrices provinciales sur le dépistage de la syphilis chez les femmes enceintes ont été révisées pour recommander plusieurs dépistages : à la première visite, entre les semaines 28 à 32 et à l'accouchement pour toutes les personnes enceintes. On recommande également un dépistage mensuel pour les personnes atteintes de syphilis nouvellement diagnostiquée qui ont été traitées pendant la grossesse en cours. De plus, les lignes directrices recommandent le dépistage d'autres infections transmissibles sexuellement et par le sang au cours du premier trimestre, notamment le $\mathrm{VIH}$, la gonorrhée, la chlamydia et l'hépatite $B$ et $C$, le cas échéant.

\section{Définitions}

\section{Tests de routine}

La date de réception par le laboratoire a été utilisée pour extraire tous les tests de syphilis du $1^{\mathrm{er}}$ janvier 2015 au 31 décembre 2019; cette date a été utilisée pour définir l'année de dépistage. Une personne a été dénombrée une fois au cours d'une année civile, le test le plus ancien ayant été utilisé comme date de référence; l'âge à la date de référence a été utilisé pour définir les groupes d'âge. Les données sur la population ont été fournies par Santé, Aînés et Vie active du Manitoba. Le taux brut de dépistage a été calculé en utilisant la population moyenne appropriée comme dénominateur; le nombre de personnes a été compté dans le numérateur. Les cas pour lesquels il manquait des renseignements sur le sexe, l'âge et la région ont été exclus des analyses (moins de $1 \%$ des cas). Le cas échéant, les taux ont été stratifiés en fonction des offices régionaux de la santé, des régions géographiques administratives établies aux fins de la prestation des soins de santé. Au Manitoba, il y a un total de cinq offices régionaux de la santé (avec les populations déclarées en 2020) (22): l'Office régional de la santé de Winnipeg (ORSW; $n=791$ 284); Southern Health-Santé Sud (SH-SS : $n=211$ 896); Santé Prairie Mountain (SPM; $n=172$ 641); l'Office régional de la santé d'Entre-les-Lacs et de l'Est (IERHA; $n=133$ 834); et l'Office régional de la santé du Nord (NRHA; $n=77283$ ).

\section{Test prénatal}

La fréquence des tests prénataux de dépistage de la syphilis était fondée sur le nombre de femmes qui accouchent chaque 
année, selon la date de l'accouchement. Pour une année donnée, le nombre de femmes testées au cours de chaque trimestre a été " calculé rétrospectivement » en fonction de la date de l'accouchement, et l'âge gestationnel figurant dans l'enregistrement de l'accouchement a servi à calculer les trimestres. Les semaines 0 à 13 ont été utilisées pour désigner le premier trimestre, les semaines 14 à 27 , le deuxième, et les semaines 28 ou plus, le troisième trimestre. Il s'agit de l'âge de la mère à la date de l'accouchement.

\section{Analyses}

Pour les analyses décrivant les tests de routine de la syphilis, les taux ont été normalisés selon l'âge pour la population canadienne à partir du Recensement de 2012; des intervalles de confiance de $95 \%$ (IC à $95 \%$ ) ont été générés (23). Les modèles de régression de Poisson, avec le logarithme de la population entré comme compensation, ont été utilisés pour produire des rapports de taux non ajustés et ajustés avec des IC à $95 \%$. Le groupe d'âge, le sexe et l'année du test ont été inclus dans les modèles de régression. Le taux de variation annuel brut a été calculé à l'aide des modèles de régression de Poisson. Pour le dépistage prénatal, le nombre de femmes au Manitoba, par office régional de la santé et groupe d'âge, a été utilisé dans le dénominateur pour les calculs selon l'âge. Les IC à $95 \%$ ont été estimés en utilisant la répartition binomiale exacte. Seules les femmes de 10 à 59 ans ont été incluses dans le numérateur et le dénominateur pour les calculs de dépistage prénatal.

\section{Résultats}

Au cours de la période de cinq ans allant de 2015 à 2019, un total de 485808 tests de syphilis ont été effectués au laboratoire provincial Cadham, ce qui représente 386735 personnes testées.
En excluant les cas pour lesquels il manque des renseignements (sur la géographie, le sexe et l'âge), un total de 475231 tests ont été effectués sur 386350 personnes. Des augmentations des tests ont été observées chaque année (tableau 1 et figure 1). En 2015, un total de 62252 personnes ont été testées (taux normalisé selon l'âge : 461,5 pour 10000 habitants [IC à $95 \%$ : 457,8-465,1]); ce nombre est passé à 94578 en 2019 (703,7 pour 10000 habitants; IC à $95 \%$ : 699,2-708,2). II s'agissait d'une augmentation annuelle de $10,5 \%$ au cours de la période à l'étude $(p<0,0001)$, tandis que le ratio annuel de dépistage homme-femme est passé de 1,8 à 1,6. Le ratio d'échantillons par rapport aux patients a augmenté en moyenne de $17 \%$ (de 1,15 à 1,35) de 2015 à 2019. La figure 2 illustre les variations des taux de tests normalisés selon l'âge par office régional de la santé au cours de la période à l'étude. Au niveau provincial, les taux ont augmenté de 2015 à 2019, mais cette augmentation était hétérogène entre les offices régionaux de la santé; le NRHA et l'ORSW affichaient les augmentations les plus importantes, tandis que les taux sont restés relativement similaires dans $\mathrm{SH}-\mathrm{SS}$ tout au long de la période d'étude.

Les résultats de la régression de Poisson multivariable ont démontré qu'après avoir ajusté toutes les autres variables du modèle, les hommes ont été testés à un taux légèrement supérieur à la moitié de celui des femmes (risque relatif ajusté [RRA] : 0,57, IC $95 \%$ : 0,56-0,57; tableau 2); le nombre de personnes testées pour la syphilis en 2019 a augmenté de $50 \%$ par rapport à 2015 (RRA : 1,52, IC $95 \%$ : 1,50-1,53); et les taux de dépistage chez les 25 à 29 ans étaient deux fois plus élevés que chez les 15 à 19 ans (RRA : 1,99, IC $95 \%$ : 1,97-2,02). Une interaction selon le sexe (non illustrée) a révélé une interaction significative entre l'année et le sexe $(p<0,0001)$, ce qui suggère que la proportion d'hommes qui ont passé un test par rapport aux femmes a augmenté au fil du temps.

Tableau 1 : Fréquence, taux normalisés selon l'âge (pour 10000 habitants) et IC à $95 \%$, tous les tests de dépistage de la syphilis effectués au laboratoire provincial de Cadham, selon le sexe, au Manitoba (2015 à 2019)a

\begin{tabular}{|c|c|c|c|c|c|c|c|c|c|c|}
\hline \multirow{3}{*}{ Determinants } & \multicolumn{3}{|c|}{ Femme } & \multicolumn{3}{|c|}{ Homme } & \multicolumn{3}{|c|}{ Total } & \multirow{3}{*}{$\begin{array}{l}\text { Rapport } \\
\text { femme- } \\
\text { homme }\end{array}$} \\
\hline & \multirow{2}{*}{ Nombre } & \multicolumn{2}{|c|}{$\begin{array}{c}\text { Standardisé selon } \\
\text { I'âge }\end{array}$} & \multirow{2}{*}{ Nombre } & \multicolumn{2}{|c|}{$\begin{array}{c}\text { Standardisé selon } \\
\text { l'âge }\end{array}$} & \multirow{2}{*}{ Nombre } & \multicolumn{2}{|c|}{$\begin{array}{c}\text { Standardisé selon } \\
\text { l'âge }\end{array}$} & \\
\hline & & Taux & IC à $95 \%$ & & Taux & IC à $95 \%$ & & Taux & IC à $95 \%$ & \\
\hline \multicolumn{11}{|l|}{ Année } \\
\hline 2015 & 40333 & 599,2 & $593,4-605,1$ & 21919 & 327,2 & $322,9-331,6$ & 62252 & 461,5 & $457,8-465,1$ & 1,83 \\
\hline 2016 & 45952 & 682,7 & $676,5-689,0$ & 24842 & 371,5 & $366,9-376,2$ & 70794 & 525,1 & $521,2-529,0$ & 1,84 \\
\hline 2017 & 48831 & 726,0 & $719,6-732,5$ & 27182 & 407,2 & $402,4-412,1$ & 76013 & 564,4 & $560,4-568,5$ & 1,78 \\
\hline 2018 & 51967 & 773,1 & $766,5-779,8$ & 30746 & 461,1 & $456,0-466,3$ & 82713 & 615,0 & $610,8-619,2$ & 1,68 \\
\hline 2019 & 58499 & 871,4 & $864,3-878,5$ & 36079 & 540,8 & $535,2-546,4$ & 94578 & 703,7 & $699,2-708,2$ & 1,61 \\
\hline Total & 245582 & 730,5 & $727,6-733,4$ & 140768 & 421,6 & $419,2-423,8$ & 386350 & 573,9 & $572,1-575,7$ & 1,73 \\
\hline $\begin{array}{l}\text { Taux de } \\
\text { changement }^{\mathrm{b}}\end{array}$ & s.o. & $9,1 \%$ & $8,8 \%-9,4 \%$ & s.o. & $13,1 \%$ & $12,6 \%-13,5 \%$ & s.o. & $10,5 \%$ & $10,3 \%-10,8 \%$ & s.o. \\
\hline
\end{tabular}

Abréviations : IC, intervalles de confiance; s.o., sans objet

Les comptages comprennent le nombre total de dépistages effectués. La population canadienne de 2012 (de Statistique Canada) a été utilisée comme population normalisée ${ }^{b}$ Calculé par la régression de Poisson à l'aide des taux bruts 
Figure 1 : Taux normalisé selon l'âge ${ }^{a}$ (pour 10000 habitants), personnes testées pour la syphilis au Manitoba, selon le sexe et l'année (2015 à 2019, $N=386350)^{b}$

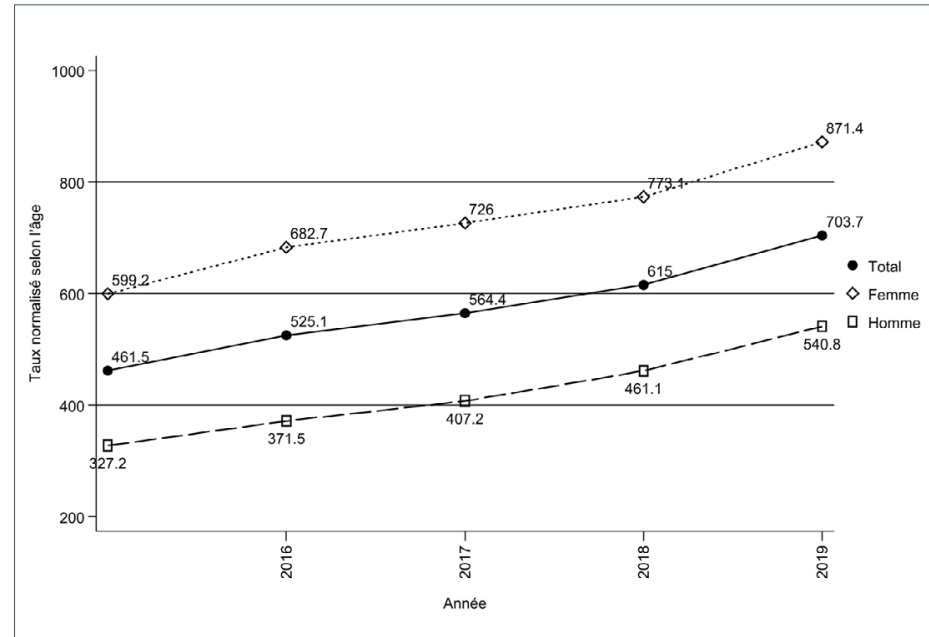

${ }^{a}$ Normalisé pour la population canadienne de 2012 (par Statistique Canada). Tous les taux sont pour 10000 habitants

${ }^{b}$ Les chiffres comprennent les personnes testées pour la syphilis cette année-là
Figure 2 : Carte thermique des taux normalisés selon l'âge (pour 10000 habitants), personnes testées pour la syphilis au Manitoba, par région sociosanitaire et année (2015 à 2019, N = 386 350)

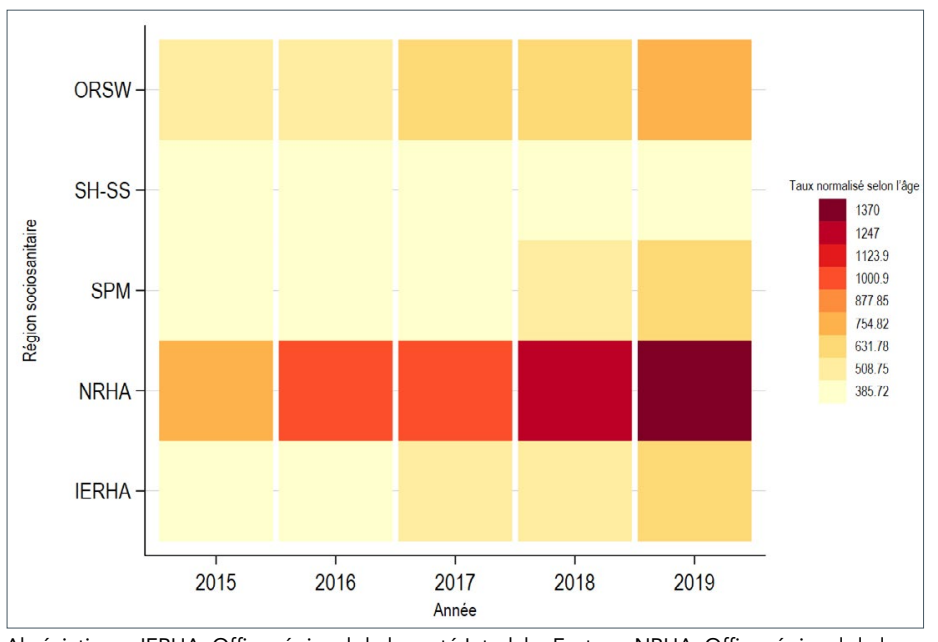

Abréviations : IERHA, Office régional de la santé Interlake-Eastern; NRHA, Office régional de la santé du Nord; ORSW, Office régional de la santé de Winnipeg; SH-SS, Southern Health-Sante Sud; SPM, Santé Prairie Mountain

Tableau 2 : Taux brut (pour 10000 habitants), risques relatifs non ajustés et ajustés et IC à $95 \%$ à partir de la régression de Poisson et déterminants du dépistage de la syphilis au Manitoba (2015 à 2019)

\begin{tabular}{|c|c|c|c|c|c|c|}
\hline Déterminants & \multicolumn{2}{|c|}{ Taux brut } & RRNA & IC à $95 \%$ & RRA & IC à $95 \%$ \\
\hline \multicolumn{7}{|l|}{ Sex } \\
\hline Femme & 719,6 & $716,7-722,2$ & Réf & s.o. & Réf & s.o. \\
\hline Homme & 417,5 & $415,3-419,7$ & 0,58 & $0,58-0,58$ & 0,57 & $0,56-0,57$ \\
\hline \multicolumn{7}{|l|}{ Année } \\
\hline 2015 & 458,8 & $455,2-462,4$ & Réf & s.o. & Réf & s.o. \\
\hline 2016 & 521,7 & $517,9-525,6$ & 1,14 & $1,13-1,15$ & 1,14 & $1,13-1,15$ \\
\hline 2017 & 560,2 & $556,2-564,2$ & 1,22 & $1,21-1,23$ & 1,22 & $1,21-1,23$ \\
\hline 2018 & 609,6 & $605,4-613,7$ & 1,33 & $1,32-1,34$ & 1,33 & $1,31-1,34$ \\
\hline 2019 & 697,0 & $692,5-701,4$ & 1,52 & $1,51-1,53$ & 1,52 & $1,50-1,53$ \\
\hline \multicolumn{7}{|l|}{ Groupe d'âge } \\
\hline Moins de 15 ans & 37,3 & $36,2-38,3$ & 0,04 & $0,04-0,05$ & 0,04 & $0,04-0,05$ \\
\hline 15 à 19 & 841,8 & $833,1-850,5$ & Réf & s.o. & Réf & s.o. \\
\hline 20 à 24 & 1491,0 & $1480,2-2022$ & 1,77 & $1,75-1,79$ & 1,77 & $1,75-1,79$ \\
\hline 25 à 29 & 1694,0 & $1682,5-1705,6$ & 2,01 & $1,99-2,04$ & 1,99 & $1,97-2,02$ \\
\hline 30 à 39 & 1208,6 & $1201,5-1215,7$ & 1,44 & $1,42-1,45$ & 1,42 & $1,40-1,44$ \\
\hline 40 à 49 & 467,8 & $463,2-472,5$ & 0,56 & $0,55-0,56$ & 0,55 & $0,54-0,56$ \\
\hline 50 ans ou plus & 168,3 & $166,7-170,0$ & 0,20 & $0,20-0,20$ & 0,20 & $0,19-0,20$ \\
\hline
\end{tabular}

Abréviations : IC, intervalles de confiance; Réf, référence; RRA, risque relatif ajusté; RRNA, risque relatif non ajusté; s.o., sans objet

Au cours de la période à l'étude, environ 77000 femmes ont passé un test prénatal de syphilis, et le nombre annuel de tests était stable à environ 15500 femmes par année (non illustré). Le tableau 3 montre les taux de dépistage prénatal selon l'âge et la région; le taux de dépistage par habitant chez les femmes de 10 à 59 ans était de 294,2 pour 10000 (IC à $95 \%: 292,2-296,3$ ) de 2015 à 2019. Les taux de dépistage étaient les plus élevés dans le NRHA (487 [IC à $95 \%$ : 476-497] pour 10000 ) et les plus faibles dans l'IERHA (262 [IC à $95 \%$ : 256-269] pour 100 000).
Il y a eu un gradient des taux selon l'âge dans l'ensemble des offices régionaux de la santé, les taux selon l'âge étant les plus élevés dans le groupe des 25 à 39 ans, à l'exception du NRHA. Au sein du NRHA, le taux le plus élevé par âge a été observé dans le groupe des 15 à 24 ans, à 1184 (IC à $95 \%$ : 1 148-1220) pour 10000 . Il s'agissait du taux de dépistage de la syphilis prénatale le plus élevé par groupe d'âge et par strate des offices régionaux de la santé dans notre étude. À l'aide d'une série de diagrammes de Venn, la figure 3 montre la répartition des tests 
prénataux, par trimestre. Jusqu'en 2018, la majorité des femmes qui ont accouché n'avaient passé qu'un seul test de dépistage prénatal de la syphilis, et ce test a été effectué au cours de leur premier trimestre; de 2015 à 2018, cette proportion variait entre $59 \%$ et $63 \%$. Au cours de cette période, environ $1 \%$ ont passé au moins un test de dépistage au cours des trois trimestres. Un changement important des tendances en matière de tests a été observé en 2019 , et seulement $39 \%$ des femmes passent maintenant un test de dépistage au cours de leur premier trimestre. La proportion de femmes ayant fait l'objet de tests au cours des premier et troisième trimestres est passée de 1,5\% en 2015 à $19 \%$ en 2019. De même, la proportion de femmes ayant passé des tests au deuxième et au troisième trimestres est passée de 1,1\% à $10 \%$ entre 2015 et 2019. Enfin, environ 3,6\% des femmes âgées de 10 à 59 ans qui ont accouché en 2019 ont passé un test de syphilis prénatal dans les trois trimestres, soit 20 fois plus qu'en 2015.

Tableau 3 : Fréquence et taux selon l'âge (pour 10 000) des tests de dépistage de la syphilis prénatale pour les femmes du Manitoba (de 10 à 59 ans), selon le groupe d'âge et l'office régional de la santé (2015 à 2019)a

\begin{tabular}{|c|c|c|c|c|c|c|c|c|c|c|c|c|c|c|c|}
\hline \multirow{2}{*}{$\begin{array}{c}\text { Groupe } \\
\text { d'âge } \\
\text { (années) }\end{array}$} & \multicolumn{3}{|c|}{ ORSW } & \multicolumn{3}{|c|}{ SPM } & \multicolumn{3}{|c|}{ IERHA } & \multicolumn{3}{|c|}{ NRHA } & \multicolumn{3}{|c|}{ SH-SS } \\
\hline & Nombre & Taux & $\begin{array}{l}\text { IC à } \\
95 \%\end{array}$ & Nombre & Taux & $\begin{array}{l}\text { IC à } \\
95 \%\end{array}$ & Nombre & Taux & $\begin{array}{l}\text { IC à } \\
95 \%\end{array}$ & Nombre & Taux & $\begin{array}{l}\text { IC à } \\
95 \%\end{array}$ & Nombre & Taux & $\begin{array}{l}\text { IC à } \\
95 \%\end{array}$ \\
\hline $\begin{array}{l}\text { Moins } \\
\text { de 15 } \\
\text { ans }\end{array}$ & 33 & 1,0 & $\begin{array}{r}0,7- \\
1,5\end{array}$ & 11 & 1,4 & $\begin{array}{r}0,7- \\
2,5\end{array}$ & 13 & 2,3 & $\begin{array}{r}1,3- \\
4,0\end{array}$ & 59 & 10,6 & $8,1-13,7$ & 13 & 1,1 & $0,6-2,0$ \\
\hline 15 à 24 & 6465 & 264,3 & $\begin{array}{r}258,0- \\
270,7 \\
\end{array}$ & 2386 & 464,5 & $\begin{array}{r}446,4- \\
483,0 \\
\end{array}$ & 1936 & 500,4 & $\begin{array}{r}478,9- \\
522,6 \\
\end{array}$ & 3595 & 1184,0 & $\begin{array}{r}1147,8- \\
1220,8 \\
\end{array}$ & 3679 & 529,0 & $\begin{array}{r}512,5- \\
545,9 \\
\end{array}$ \\
\hline 25 à 39 & 32912 & 766,2 & $\begin{array}{r}758,2- \\
774,2\end{array}$ & 6704 & 819,0 & $\begin{array}{r}800,3- \\
838,0\end{array}$ & 4155 & 782,3 & $\begin{array}{r}759,6- \\
805,5\end{array}$ & 4387 & 1107,1 & $\begin{array}{r}1076,4- \\
1138,4\end{array}$ & 9289 & 945,9 & $\begin{array}{r}927,6- \\
964,3\end{array}$ \\
\hline \begin{tabular}{|l|}
40 ans \\
ou plus
\end{tabular} & 1107 & 21,5 & $\begin{array}{r}20,3- \\
22,8 \\
\end{array}$ & 152 & 14,5 & $\begin{array}{r}12,3- \\
17,0 \\
\end{array}$ & 101 & 11,3 & $\begin{array}{l}9,2- \\
13,7\end{array}$ & 101 & 24,2 & $\begin{array}{r}19,7- \\
29,4 \\
\end{array}$ & 275 & 22,8 & $\begin{array}{r}20,2- \\
25,6 \\
\end{array}$ \\
\hline Total & 40517 & 268,9 & $\begin{array}{r}266,3- \\
271,5\end{array}$ & 9253 & 292,0 & $\begin{array}{r}286,2- \\
297,9\end{array}$ & 6205 & 262,2 & $\begin{array}{r}255,8- \\
268,7\end{array}$ & 8142 & 486,5 & $\begin{array}{r}476,2- \\
496,9\end{array}$ & 13256 & 329,7 & $\begin{array}{r}324,2- \\
335,3\end{array}$ \\
\hline
\end{tabular}

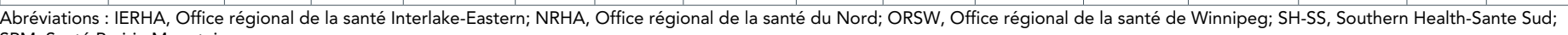
SPM, Santé Prairie Mountain

a Les chiffres comprennent les personnes qui ont fait l'objet d'un dépistage de la syphilis cette année-là

Figure 3 : Tendances du dépistage prénatal de la syphilis, selon le trimestre et l'année de la naissance de l'enfant pour les femmes du Manitoba, 2015 à 2019

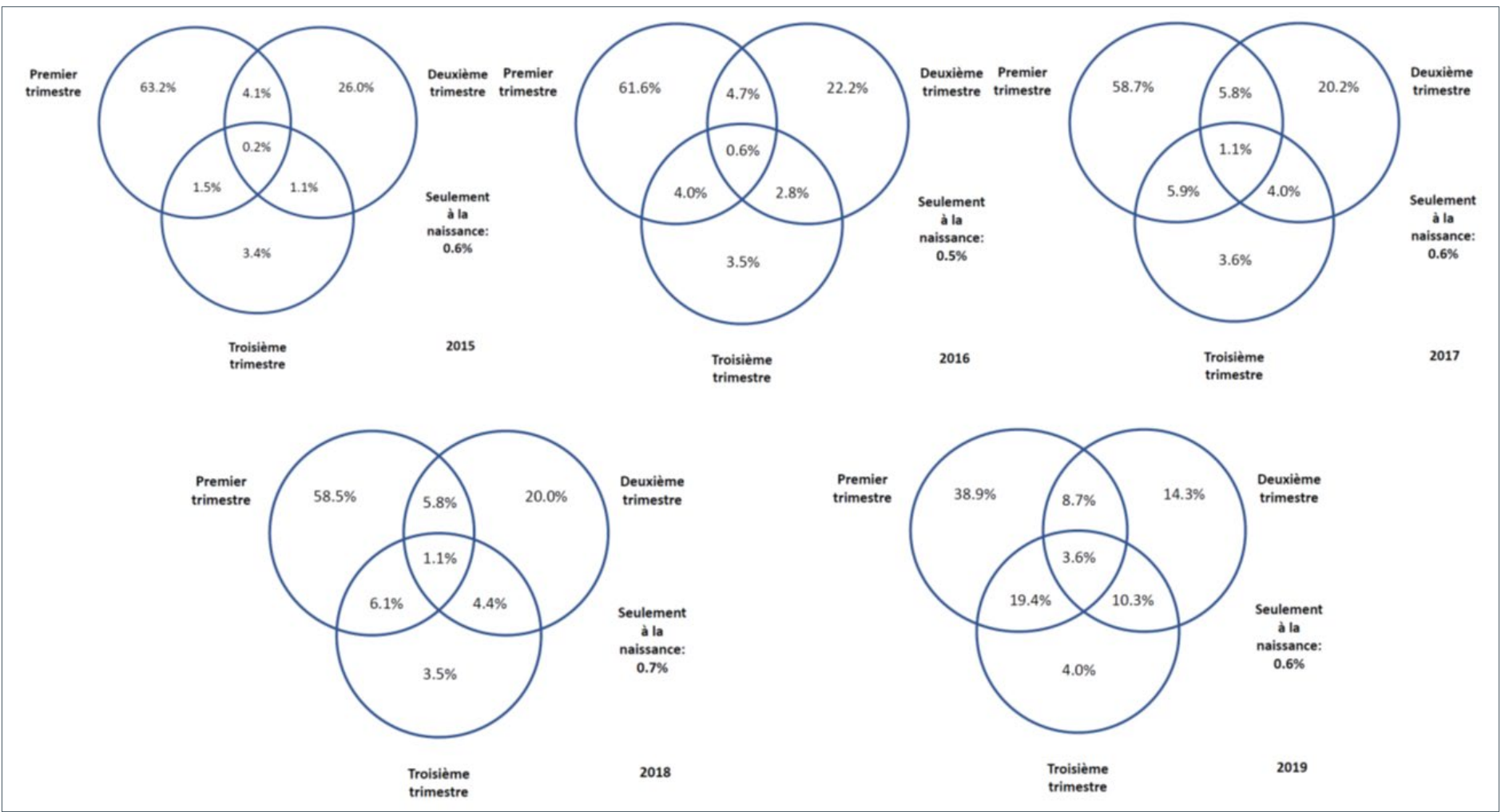




\section{Discussion}

Selon l'Agence de la santé publique du Canada, il y a eu une augmentation de $153 \%$ des taux de syphilis infectieuse entre 2014 et 2018 au Canada. Toutefois, le Manitoba a affiché une augmentation de $560 \%$ au cours de la même période (4). Selon une étude récente, il y a eu une augmentation des taux de syphilis chez les femmes de Winnipeg depuis 2014, et des éclosions chez les jeunes femmes sont associées à la vie au centre-ville, à la consommation de substances et à la co-infection à la chlamydia (5). Au moment de l'étude, $24 \%$ des femmes ont déclaré être enceintes, toutefois aucun cas de syphilis congénitale n'a été détecté (5). À la suite de cette étude, le Manitoba a signalé son premier cas de syphilis congénitale en plus de 50 ans en 2015, et un autre cas a été signalé en 2017 (4). Le nombre de cas de syphilis congénitale au Manitoba a augmenté considérablement depuis, avec au moins 30 cas signalés en 2020 à Winnipeg seulement, pour un taux brut de 3,5 pour 1000 naissances vivantes (13).

Santé, Aînés et Vie active du Manitoba a recommandé que toutes les femmes enceintes se soumettent à un dépistage de la syphilis au cours de leur première visite prénatale (24); toutefois, la transmission verticale de la syphilis peut se produire malgré les programmes de dépistage prénatal existants étant donné que les femmes peuvent être infectées entre le moment du test et l'accouchement et que les cliniciens peuvent omettre de diagnostiquer la syphilis en raison de manifestations cliniques non spécifiques $(12,14)$. Nos résultats montrent un changement récent et important dans la pratique du dépistage prénatal. En effet, la proportion de femmes qui ont passé un seul test pendant la grossesse est passée de $93 \%$ en 2015 à $57 \%$ en 2019. À l'inverse, la proportion de femmes ayant passé deux tests ou plus est passée de $7 \%$ en 2015 à $43 \%$ en 2019. Compte tenu de l'épidémie croissante de syphilis au Manitoba, une augmentation encore plus importante des taux de dépistage au troisième trimestre pourrait mener à la détection d'un plus grand nombre de cas de syphilis congénitale. La proportion persistante de femmes dont le dossier de santé indique que le seul test de dépistage prénatal qu'elles ont reçu était au moment de l'accouchement est préoccupante. Cette proportion variait de $0,5 \%$ à $0,7 \%$ au cours d'une année donnée et, au cours de la période d'étude, touchait environ 400 femmes. De plus, des données récentes laissent entendre que la pandémie de COVID-19 a une incidence importante sur les taux de dépistage et les visites à la clinique pour les infections transmissibles sexuellement et par le sang en Amérique du Nord (25-27). Dans le cas de la syphilis, cette situation peut avoir entraîné une augmentation de la proportion de syphilis non diagnostiquée, ce qui a entraîné une augmentation de la syphilis congénitale. La surveillance des taux de dépistage prénatal de la syphilis est donc essentielle à l'élimination de la syphilis congénitale. Les Centres pour le contrôle et la prévention des maladies des États-Unis ont suggéré d'offrir des tests de dépistage selon diverses modalités, notamment avec des cliniques sans rendez-vous, des services de télésanté et au moyen de trousses d'autodépistage (28). Compte tenu de la disponibilité des données de dépistage fondées sur la population au Manitoba, les recherches futures devraient explorer l'incidence de la pandémie de COVID-19 sur les taux de dépistage de la syphilis et d'autres infections transmissibles sexuellement et par le sang, et décrire si certaines sous-populations étaient plus susceptibles d'être touchées. La propagation de la syphilis à la population hétérosexuelle (qui touchait majoritaire la population gbHARSAH par le passé) a probablement joué un rôle dans la diminution du ratio femme-homme, les hommes gbHARSAH et hétérosexuels étant plus susceptibles de subir un test de dépistage de la syphilis. Cela a été confirmé par le fait que l'interaction sexuelle était statistiquement significative dans les modèles de régression. La surveillance des tendances de tests de dépistage de la syphilis chez les hommes avant et après l'arrivée de la COVID-19 sera un important objectif de surveillance.

En plus de la syphilis congénitale, la syphilis infectieuse a pour autre conséquence de provoquer la neurosyphilis. L'augmentation du nombre d'infections par la syphilis au Manitoba devrait entraîner une augmentation du nombre de cas de neurosyphilis. Un examen des cas de neurosyphilis en Alberta, au Canada, a révélé environ 30 cas de neurosyphilis au stade précoce et tardif chaque année entre 2015 et 2016 (7). Les données du programme du laboratoire provincial Cadham ont signalé 23 et 28 cas de neurosyphilis détectés en laboratoire (jusqu'en septembre 2019) en 2018 et 2019, respectivement, $d$ 'après des tests de laboratoire de recherche sur le liquide céphalorachidien réactif du Laboratoire de recherche sur les maladies vénériennes effectués avec du liquide céphalorachidien non sanglant (Stein, communication personnelle). Landry et al. ont constaté des différences sociodémographiques claires entre les personnes diagnostiquées d'une neurosyphilis précoce et tardive (7). Les cas de neurosyphilis précoce étaient plus susceptibles d'être des hommes, nés au Canada, de race blanche et qui ont déclaré avoir des relations sexuelles avec d'autres hommes; en revanche, les cas de neurosyphilis tardive étaient également plus susceptibles d'être des hommes, mais d'être nés à l'extérieur du Canada et de s'identifier comme hétérosexuels (7). II est fortement recommandé que les futurs efforts de surveillance visent à détecter la neurosyphilis.

\section{Forces et faiblesses}

L'une des forces de notre étude était qu'elle était fondée sur les données de la population; par conséquent, les taux de dépistage n'étaient pas limités à certaines sous-populations. Les limites comprennent des renseignements démographiques, cliniques et épidémiologiques limités; une recherche plus poussée devrait relier les personnes à d'autres ensembles de données administratives sur les soins de santé. En raison des limites de l'accès aux taux incidents de syphilis, les taux de dépistage n'ont pas été comparés à l'incidence de la syphilis par office régional de la santé; cependant, le NRHA et l'ORSW ont toujours déclaré les taux les plus élevés de syphilis infectieuse (29). 


\section{Conclusion}

Nos résultats montrent que le nombre de personnes qui se soumettent à des tests de dépistage de la syphilis a augmenté entre 2015 et 2019. Au Manitoba, I'incidence croissante de la syphilis infectieuse a fait l'objet d'une exposition médiatique importante, et la santé publique a alerté les praticiens de la nécessité du dépistage de la syphilis (4). Les médias et les communications de santé publique ont probablement contribué à l'augmentation des taux de dépistage. Les recherches à venir devraient déterminer si les tests et le dépistage atteignent les populations les plus appropriées, surtout compte tenu de l'augmentation des cas de syphilis congénitale observés à Winnipeg.

\section{Déclaration des auteurs}

S. Y. S. - Analyses conceptuelles, analyse statistique et rédaction de la version préliminaire de l'article

P. J. P. - Interprétation des constatations et révision critique de I'article pour le contenu intellectuel

P. K. - Conception du processus d'extraction, acquisition des données, réalisation des analyses statistiques initiales et contribution aux révisions de l'article

D. S. - Analyses conceptuelles, interprétation des constatations et révision de l'article de façon critique pour le contenu intellectuel

Chaque auteur répondait aux critères de l'International Committee of Medical Journal Editors. Tous les auteurs ont approuvé la version finale de l'article.

Le contenu de l'article et les points de vue qui y sont exprimés n'engagent que les auteurs et ne correspondent pas nécessairement à ceux du gouvernement du Canada.

\section{Intérêts concurrents}

Aucun

\section{Remerciements}

Aucun.

\section{Financement}

S. Y. S. était appuyé par une chaire de recherche du Canada en science des programmes et en santé publique mondiale (niveau II).

\section{Références}

1. Choudhri Y, Miller J, Sandhu J, Leon A, Aho J. La syphilis infectieuse et la syphilis congénitale au Canada, de 2010 à 2015. Relevé des maladies transmissibles au Canada. 2018;44(2):47-53. DOI

2. Centers for Disease Control and Prevention. Sexually Transmitted Disease Surveillance 2018. Atlanta (GA): CDC; 2019. https://www.cdc.gov/std/stats18/ STDSurveillance2018-full-report.pdf

3. Agence de la santé publique du Canada. Rapport sur les infections transmissibles sexuellement au Canada, 2017. Ottawa (ON) : ASPC; 2019. https://www.canada.ca/fr/ sante-publique/services/publications/maladies-et-affections/ rapport-infections-transmissibles-sexuellement-canada-2017. html

4. Agence de la santé publique du Canada. La syphilis au Canada: rapport technique sur les tendances épidémiologiques, les déterminants et interventions. Ottawa (ON) : ASPC; 2020. https://www.canada.ca/fr/services/ sante/publications/maladies-et-affections/syphilis-rapportepidemiologiques.html

5. Shaw SY, Ross C, Nowicki DL, Marshall S, Stephen S, Davies C, Riddell J, Bailey K, Elliott LJ, Reimer JN, Plourde PJ. Infectious syphilis in women: what's old is new again? Int J STD AIDS 2017;28(1):77-87. DOI PubMed

6. Kidd SE, Grey JA, Torrone EA, Weinstock HS. Increased Methamphetamine, Injection Drug, and Heroin Use Among Women and Heterosexual Men with Primary and Secondary Syphilis - United States, 2013-2017. Morb Mortal Wkly Rep 2019;68(6):144-8. DOl PubMed

7. Landry T, Smyczek P, Cooper R, Gratrix J, Bertholet L, Read R, Romanowski B, Singh AE. Retrospective review of tertiary and neurosyphilis cases in Alberta, 1973-2017. BMJ Open 2019;9(6):e025995. DOI PubMed

8. Kamb ML, Newman LM, Riley PL, Mark J, Hawkes SJ, Malik T, Broutet N. A road map for the global elimination of congenital syphilis. Obstet Gynecol Int 2010;2010:312798. DOl PubMed

9. Shahrook S, Mori R, Ochirbat T, Gomi H. Strategies of testing for syphilis during pregnancy. Cochrane Database Syst Rev 2014;10(10):CD010385. DOI PubMed

10. Organisation mondiale de la Santé. L'élimination mondiale de la syphilis congénitale : raison d'être et stratégie. Genève (CH) : OMS; 2007. https://www.who.int/reproductivehealth/ publications/rtis/9789241595858/fr/

11. ProMED. Syphilis - USA (09): (CA) pregnant women, congenital, rising incidence. Archive \#20150715.3512635. July 14, 2015. http://www.promedmail.org/ post/20150715.3512635 
12. Keuning MW, Kamp GA, Schonenberg-Meinema D, Dorigo-Zetsma JW, van Zuiden JM, Pajkrt D. Congenital syphilis, the great imitator-case report and review. Lancet Infect Dis 2020;20(7):e173-9. DOI PubMed

13. Benoit P, Tennenhouse LG, Lapple A, Hill-Carroll G, Shaw SY, Bullard J, Plourde P. Résurgence de la syphilis congénitale à Winnipeg, Manitoba. Relevé des maladies transmissibles au Canada 2022;48(2/3):107-14. DOI

14. Lin JS, Eder ML, Bean SI. Screening for Syphilis Infection in Pregnant Women: Updated Evidence Report and Systematic Review for the US Preventive Services Task Force. JAMA 2018;320(9):918-25. DOI PubMed

15. U.S. Preventive Services Task Force. Screening for syphilis infection in pregnancy: U.S. Preventive Services Task Force reaffirmation recommendation statement. Ann Intern Med 2009;150(10):705-9. DOl PubMed

16. Bibbins-Domingo K, Grossman DC, Curry SJ, Davidson KW, Epling JW Jr, García FA, Gillman MW, Harper DM, Kemper AR, Krist AH, Kurth AE, Landefeld CS, Mangione CM, Phillips WR, Phipps MG, Pignone MP; US Preventive Services Task Force (USPSTF). Screening for Syphilis Infection in Nonpregnant Adults and Adolescents: US Preventive Services Task Force Recommendation Statement. JAMA 2016;315(21):2321-7. DOI PubMed

17. Tuite AR, Shaw S, Reimer JN, Ross CP, Fisman DN, Mishra S. Can enhanced screening of men with a history of prior syphilis infection stem the epidemic in men who have sex with men? A mathematical modelling study. Sex Transm Infect 2018;94(2):105-10. DOI PubMed

18. Tuite A, Fisman D. Go big or go home: impact of screening coverage on syphilis infection dynamics. Sex Transm Infect 2016;92(1):49-54. DOl PubMed

19. Neblett Fanfair R, Tao G, Owusu-Edusei K, Gift TL, Bernstein KT. Suboptimal Prenatal Syphilis Testing Among Commercially Insured Women in the United States, 2013. Sex Transm Dis 2017;44(4):219-21. DOI PubMed

20. CBC News. 10 infants infected with syphilis as outbreak worsens. Feb 25, 2019. https://www.cbc.ca/news/canada/ manitoba/syphilis-manitoba-outbreak-infants-1.5032422

21. Lix L, Smith M, Azimaee M, Dahl M, Nicol P, Burchill C, Burland E, Goh C, Schultz J, Bailly A. A Systematic Investigation of Manitoba's Provincial Laboratory Data. Winnipeg (MB): MCHP; 2012. http://mchp-appserv.cpe. umanitoba.ca/landing.php?referencePaperID=76804
22. Manitoba Health Seniors and Active Living. Population Report, June 1, 2020. Winnipeg (MB): Government of Manitoba; 2020. https://www.gov.mb.ca/health/population/ pr2020.pdf

23. Tiwari RC, Clegg LX, Zou Z. Efficient interval estimation for age-adjusted cancer rates. Stat Methods Med Res 2006;15(6):547-69. DOI PubMed

24. Manitoba Health Seniors Care. Updates to Syphilis Protocol Congenital Syphilis and Syphilitic Stillbirth Case Definitions and Reporting. Winnipeg (MB): Government of Manitoba; Oct 12, 2021. https://www.gov.mb.ca/health/publichealth/ cdc/protocol/syphilis.pdf

25. Menza TW, Zlot Al, Garai J, Humphrey S, Ferrer J. The Impact of the SARS-CoV-2 Pandemic on Human Immunodeficiency Virus and Bacterial Sexually Transmitted Infection Testing and Diagnosis in Oregon. Sex Transm Dis 2021;48(5):e59-63. DOI PubMed

26. Tao J, Napoleon SC, Maynard MA, Almonte A, Silva E, Toma E, Chu CT, Cormier K, Strong S, Chan PA. Impact of the COVID-19 Pandemic on Sexually Transmitted Infection Clinic Visits. Sex Transm Dis 2021;48(1):e5-7. DOI PubMed

27. Bonett S, Petsis D, Dowshen N, Bauermeister J, Wood SM. The Impact of the COVID-19 Pandemic on Sexually Transmitted Infection/Human Immunodeficiency Virus Testing Among Adolescents in a Large Pediatric Primary Care Network. Sex Transm Dis 2021;48(7):e91-3. DOI PubMed

28. Rogers B, Tao J, Murphy M, Chan PA. The COVID-19 Pandemic and Sexually Transmitted Infections: Where Do We Go From Here? Sex Transm Dis 2021;48(7):e94-6. DOI PubMed

29. Manitoba Health Seniors and Active Living. Sexually Transmitted Infections in Manitoba: 2014. Winnipeg (MB): Government of Manitoba; November 2016. https://www. gov.mb.ca/health/publichealth/surveillance/docs/stim2014. pdf 